\title{
The Threshold Percolation Volume Fraction of Vitreous Silica on the Thermal Expansion Behavior of Quartz
}

\author{
José Antonio Alves Júnior, João Baptista Baldo \\ The Federal University of Sao Carlos (UFSCar), Materials Engineering Department (DEMa), São Carlos, SP, Brazil \\ Email: jjunior8404@gmail.com,baldo@ufscar.br
}

How to cite this paper: Alves Júnior, J.A. and Baldo, J.B. (2019) The Threshold Percolation Volume Fraction of Vitreous Silica on the Thermal Expansion Behavior of Quartz. Journal of Materials Science and Chemical Engineering, 7, 1-6.

https://doi.org/10.4236/msce.2019.76001

Received: April 27, 2019

Accepted: June 17, 2019

Published: June 20, 2019

Copyright $\odot 2019$ by author(s) and Scientific Research Publishing Inc. This work is licensed under the Creative Commons Attribution International License (CC BY 4.0). http://creativecommons.org/licenses/by/4.0/

\begin{abstract}
In this work, it is shown how the additions of vitreous silica of particle size $<100$ mesh may influence the reversible thermal expansion behavior of a particular mix of alfaquartz particles of different size distribution. It was found that the addition of $30 \approx 35$ vol\% of vitreous silica can be considered as a percolation threshold mass-volume fraction limit, which is enough to eliminate totally the characteristic thermal expansion behavior of quartz in the range from room temperature to $1100^{\circ} \mathrm{C}$.
\end{abstract}

\section{Keywords}

Quartz, Vitreous Silica, Thermal Expansion Behavior, Percolation Threshold

\section{Introduction}

Because of the polymorphism related to the alfa/beta phase transitions of crystalline quartz, tridimite and cristobalite, either during heating up or cooling down, an overall peculiar thermal expansion behavior is presented by silica. The sudden and strong expansion/contraction, in the range from $570^{\circ} \mathrm{C}$ to $600^{\circ} \mathrm{C}$ characteristic of the alfa to beta quartz transition (or the other way around), may cause microstructural weakening of quartz containing ceramics [1]. This is the case for foundry masses and masses of the triaxial class products such as porcelain, sanitary and table ware and floor and wall tile when they are heated up or cooled down even under low rates of temperature change. The necessity to control properties such as thermal shock resistance and dimensional changes of fired pieces makes it interesting to know how to manipulate the reversible thermal expansion of materials for desired macro effects.

Considering that ceramic mixes are composite materials, the formal treatment 
of the thermal expansion behavior of two-phase ceramic composites has been made using three models [2]:

1) The Rule of Mixtures

$$
\alpha_{c}=\alpha_{m} V_{m}+\alpha_{p} V_{p}
$$

2) Turner's Model

$$
\alpha_{c}=\left(\frac{\alpha_{m} V_{m} E_{m}+\alpha_{p} V_{p} E_{p}}{E_{m} V_{m}+E_{p} V_{p}}\right)
$$

3) Kerner's Model

$$
\alpha_{c}=\alpha_{m} V_{m}+\alpha_{p} V_{p}-\left(\alpha_{m}-\alpha_{p}\right) V_{m} V_{p}\left(\frac{E_{m}^{-1}-E_{p}^{-1}}{\frac{V_{p}}{E_{m}}+\frac{V_{m}}{E_{p}}+\frac{3}{4 G_{m}}}\right)
$$

In the above formulas, $\alpha$ is the linear thermal expansion coefficient, $V$ is the volumetric fraction, $E$ is the elastic modulus, $G$ is the shear modulus. The subscripts $\mathcal{C}, \mathrm{m}$ and $\mathrm{p}$ refer respectively to the composite, the matrix and the particulate inclusion.

The rule of mixtures model is applicable for the case of no interaction between the composite phases. The Turner's model is applicable when only microstresses occur under isostatic condition between the composite phases. Conversely, the Kerner's model is applicable in the case of shear and micro-isostatic stresses.

What is more evident in the above models, is that the properties of composite materials may change as a function of the volumetric fractions of their constituents as well as on their thermal and elastic properties.

Instead of using the presented models, the averaged properties in composite materials, are currently been treated in the literature under probabilistic terms using what is known as "Percolation Theory". In this subject, the aim is to find a critical valued parameter (concentration of a second phase for example) in a composite system, at which a specific system property drastically changes [3] [4].

Our interest in the present study is to investigate in a simple manner, if there is a volumetric fraction of vitreous silica addition to a mixture of differently sized quartz particles, at which the behavior of the reversible linear thermal expansion of the original system, drastically changes. If this critical vitreous silica volumetric fraction is reached, then we may consider it as a volumetric fraction percolation threshold for the drastic change in the thermal expansion behavior of quartz.

\section{Materials and Methods}

In order to homogenize at least chemically the system, in this study, silica was used in its crystalline form of $\alpha$-quartz and vitreous silica (fused quartz). In this sense, a two-phases composite was made by mixing silica materials. In this composite, the chemical reactivity between the components could be disre- 
garded and only the physical and thermal behavior of each component would come into play.

A starting composition called Mix 1 was made by mixing several high purity quartz powders of different grain size distributions. This was elected as the single phased composite whose thermal expansion behavior was set under scrutiny. To the M1 composition, additions of 100 mesh vitreous silica powder were made in increasing amounts of 5, 10, 20 and $30 \mathrm{wt} \%$, making a total of four two-phase composites (M2, M3, M4 and M5). Vitreous silica possesses extremely low coefficient of thermal expansion. This behavior has been credited to two opposite tendencies. The first is the natural increase in the silicon-oxygen bond length with increasing temperature. The second is the decrease in the bond angle at the oxygen atom, which (if great enough) can be responsible for the shrinkage. The combination of these antagonistic effects leads to the overall very low thermal expansion coefficient of vitreous silica [5].

In Table 1 the chemical composition of the used quartz.

In Table 1 the proportions five compositions investigated is presented.

For dilatometries measurements, the five different compositions were casting in a nylon bar shaped molds with dimensions of $1 \times 1 \times 5 \mathrm{~cm}$. Colloidal silica (15 wt\% liquid) was used as the binder. After demolding, they were dried at $110^{\circ} \mathrm{C} / 24$ hours.

The dilatometry tests were made in an Orton Dilatometer, from room temperature to $1050^{\circ} \mathrm{C}$ using a heating rate of $3^{\circ} \mathrm{C} /$ minute. The samples were only dried.

Methodology for determination of particle size:

When a beam of light strikes a colloidal dispersion, the suspended particles (or droplets) spread the incident radiation. The intensity of scattered light undergoes fluctuations that can be correlated with the random (Brownian) of these particles, in turn, depends on their dimensions. The equipment used for measurement was Microtrac S3500 Particle Size Analyzer.

\section{Results and Discussion}

In Figure 1 it is shown the respective particle size distributions of the materials used. It can be seen the particle size distribution of the initial M1 composition is much finer than that corresponding to vitreous silica one. This was purposely chosen in order to decrease the surface area ratio of vitreous silica to quartz mix,

Table 1. Compositions investigated in the present study.

\begin{tabular}{ccccccc}
\hline Material & D $_{50}$ & M1 & M2 & M3 & M4 & M5 \\
\hline Quartz \#200 & $13 \mu \mathrm{m}$ & $52 \mathrm{wt} \%$ & $49.4 \mathrm{wt} \%$ & $46.8 \mathrm{wt} \%$ & $41.6 \mathrm{wt} \%$ & $36.4 \mathrm{wt} \%$ \\
Quartz \#325 & $7 \mu \mathrm{m}$ & $32 \mathrm{wt} \%$ & $30.4 \mathrm{wt} \%$ & $28.8 \mathrm{wt} \%$ & $25.6 \mathrm{wt} \%$ & $22.4 \mathrm{wt} \%$ \\
Quartz \#500 & $4 \mu \mathrm{m}$ & $16 \mathrm{wt} \%$ & $15.2 \mathrm{wt} \%$ & $14.4 \mathrm{wt} \%$ & $12.8 \mathrm{wt} \%$ & $11.2 \mathrm{wt} \%$ \\
VS \#100 & $30 \mu \mathrm{m}$ & - & $5.0 \mathrm{wt} \%$ & $10.0 \mathrm{wt} \%$ & $20.0 \mathrm{wt} \%$ & $30.0 \mathrm{wt} \%$
\end{tabular}

VS = Vitreous Silica. 


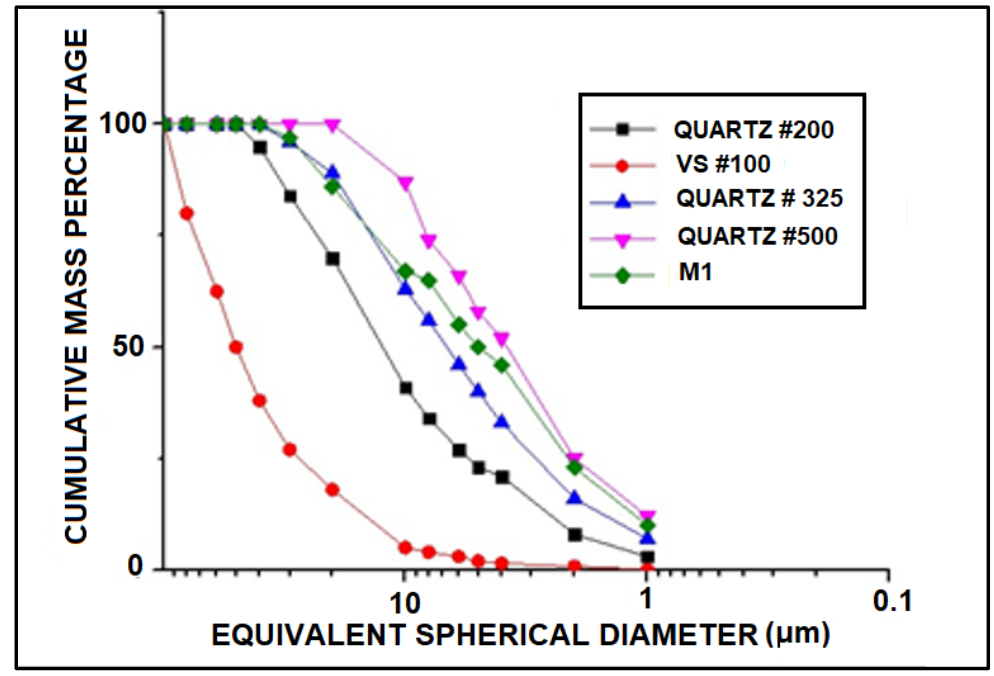

Figure 1. The particle size distribution of initial mix M1 and the raw materials used in the other four compositions.

making it difficult the creation of a continuous phase tunneling through the M1 composition spatial microstructure and also decreasing chances of reaction between quartz and vitreous silica particles.

In Figure 2 the respective linear thermal expansion behavior of the five compositions are shown. It can be seen that the behavior of composition M1 (without vitreous silica) is typical of crystalline quartz, characterized by the steep linear expansion in the range from $570^{\circ} \mathrm{C}$ to $610^{\circ} \mathrm{C}$ reaching a maximum of $1.05 \%$. We can also notice a second slight apparent increase in expansion starting around $850^{\circ} \mathrm{C}$, characteristic of the $\beta$-quartz to $\beta$-tridymite transition $(\approx 12$ vol\%) This particular phase transition is only possible in the presence of liquid phase. Exactly this liquid phase is responsible for the shrinkage that follows when the temperature gets bigger than $900^{\circ} \mathrm{C}$.

Upon the addition of vitreous silica amounts of 5 and $10 \mathrm{wt} \%$, there is an overall gradual decrease in the maximum linear expansion, maintaining however the same tendencies, resembling smaller scales of the expansion versus temperature plot of composition M1. The phase transition $\alpha$-quartz to $\beta$-quartz is still quite visible in M2 (5 wt\% VS) and M3 (10 wt\% VS) but with smaller intensity.

By adding $20 \mathrm{wt} \%$ of vitreous silica (composition M4), there is an expressive decrease in the maximum linear expansion as well as a decrease in the height in the pic related to the phase transition $\alpha$-quartz to $\beta$-quartz. In addition, there is no noticeable expansion variation for temperatures bigger than $600^{\circ} \mathrm{C}$.

Finally the addition of $30 \mathrm{wt} \%$ ( $\approx 35 \mathrm{vol} \%$ ) of vitreous silica (composition M5) promoted a drastic change in the thermal expansion behavior, as if it was composed of $100 \mathrm{wt} \%$ vitreous silica alone (100 wt $\% \mathrm{VS}$ ) as shown in the superposition of M5 (30 wt\% VS) and $100 \mathrm{wt} \%$ VS plots of Figure 2.

This last occurrence indicates that there is a critical mass fraction (or volume fraction) which indicates a concentration percolation limit of vitreous silica in the quartz mix, which is enough to eliminate the characteristic thermal expansion 


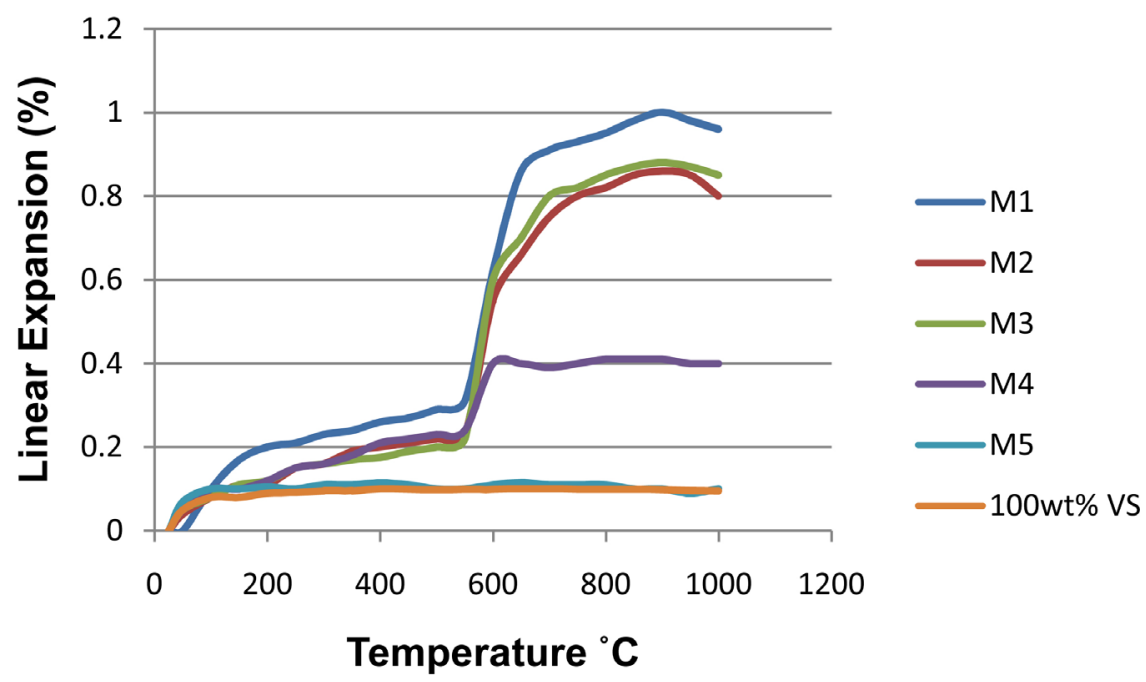

Figure 2. The linear thermal expansion behavior of the five compositions and the \#100 mesh vitreous silica alone. VS = Vitreous Silica.

behavior of the original quartz mix. The vitreous silica content of $30 \mathrm{wt} \%(\approx 35$ vol\%) clearly indicates a percolation limit.

\section{Conclusions}

The results lead to the conclusion that it is possible to drastically change (if not eliminate) the thermal expansion behavior of quartz by adding $35 \mathrm{vol} \%$ of vitreous silica in a mixture made of quartz particles of different grain size distribution.

\section{Acknowledgements}

The authors would like to thank funding agencies FAPESP (CDMF/CEPID) and Capes.

\section{Conflicts of Interest}

The authors declare no conflicts of interest regarding the publication of this paper.

\section{References}

[1] Goswami, G., Sanu, P. and Panigrahy, P.K. (2015) Estimation of Thermal Expansion of Silica Refractory Based on Its Mineralogy. Interceram-International Ceramic Review, 64, 174-176. https://doi.org/10.1007/BF03401118

[2] Karch, C. (2014) Micromechanical Analysis of Thermal Expansion Coefficients. Modeling and Numerical Simulation of Material Science, 4, 104-118. https://doi.org/10.4236/mnsms.2014.43012

[3] Stauffer, D. and Aharony, A. (1994) Introduction to Percolation Theory. 2nd Edition, Taylor \& Francis, London.

[4] Berkowitz, B., Ewing, R.P. (1998) Percolation Theory and Network Modeling Applications in Soil Physics. Surveys in Geophysics, 19, 23-72. 
https://doi.org/10.1023/A:1006590500229

[5] Gaskell, P.H. (1996) Thermal Properties of Silica. Part 2-Thermal Expansion Coefficient of Vitreous Silica. Transactions of the Faraday Society, 62, 1505-1510.

https://doi.org/10.1039/TF9666201505

\section{Explain Percolation Word}

Percolation theory is the simplest not exactly solved model displaying a phase transition. Often, the insight into the percolation theory problem facilitates the understanding of many other physical systems. Moreover, the concept of fractals, which is intimately related to the percolation theory problem, is of general interest as it pops up more or less everywhere in Nature. The knowledge of percolation, fractals, and scaling is of immense importance theoretically in such diverse fields as biology, physics, and geophysics and also of practical importance in e.g. oil recovery. We will begin gently by developing a basic understanding of percolation theory, providing a natural introduction to the concept of scaling and renormalisation group theory. 